\title{
Avaliação dos princípios da orientação familiar e comunitária da atenção primária à saúde da criança
}

\section{Evaluation of the principles of family counseling and community primary care child health}

\author{
Jéssica Mendes Daschevi ${ }^{1}$; Mauren Teresa Grubisich Mendes Tacla²; Barbara de \\ Andrade Alves ${ }^{3}$; Beatriz Rosana Gonçalves de Oliveira Toso ${ }^{4}$; Neusa Collet ${ }^{5}$
}

\begin{abstract}
Resumo
A atenção primária é considerada o primeiro contato dos indivíduos, suas famílias e a população com o sistema de saúde. Conforme se aproxima dos indivíduos e da comunidade, consegue aumentar seu poder de interação com a população. Possui quatro atributos essenciais: acesso de primeiro contato, longitudinalidade, integralidade e coordenação e três derivados: orientação familiar, orientação comunitária e competência cultural. A orientação familiar consiste no conhecimento dos fatores relacionados à origem e aos cuidados das enfermidades e a orientação comunitária reconhece as necessidades na comunidade por meio de dados epidemiológicos e contato direto. No presente estudo, o objetivo foi avaliar os princípios da orientação familiar e comunitária da atenção primária à saúde da criança em unidades básicas de saúde de Londrina, Paraná. Pesquisa descritiva de natureza quantitativa, realizada em 39 unidades básicas da área urbana, entre agosto de 2012 e janeiro de 2013 por meio de um instrumento de avaliação denominado Primary Care Assessment Tool-Brasil versão criança. Foram realizadas 609 entrevistas, respondidas pelos pais ou principais cuidadores de crianças menores de doze anos, que utilizaram o serviço no mínimo três vezes para consultas com pediatra ou enfermeiro. $\mathrm{O}$ valor do escore para a orientação familiar foi 5,082 e para orientação comunitária de 5,462, não atingindo o escore ideal $(6,6)$. Conclui-se então que ambos os atributos precisam ser desenvolvidos, com o aperfeiçoamento ou instalação de novas medidas que possam ajudar na melhoria da qualidade da atenção básica à saúde da criança.
\end{abstract}

Palavras-chave: Avaliação de serviços de saúde. Atenção primaria à saúde. Serviços de saúde.

\begin{abstract}
The primary attention is considered the first contact of individuals, their families and the population with the health system. As you approach the individuals and the community, your power to interact with the population can increase. It features four key attributes: first contact access, longitudinality, comprehensiveness and coordination, and three derivatives: family counseling, community orientation, and cultural competence. The family orientation consists in the knowledge of family factors related to the
\end{abstract}

\footnotetext{
${ }^{1}$ Aluna do Curso de Graduação em Enfermagem da Universidade Estadual de Londrina. Bolsista de Iniciação Científica. E-mail: jessica_daschevi@hotmail.com

${ }^{2}$ Enfermeira. Doutora em Enfermagem em Saúde Pública pela Escola de Enfermagem de Ribeirão Preto da Universidade de São Paulo - EERP/USP. Professor Adjunto do Curso de Enfermagem e do Mestrado em Enfermagem da Universidade Estadual de Londrina. E-mail: maurentacla@gmail.com

${ }^{3}$ Enfermeira. Mestre em Enfermagem pela Universidade Estadual de Londrina. Especialista em Saúde da Criança pela Universidade Estadual de Londrina. E-mail: andradealves.b@gmail.com

${ }^{4}$ Enfermeira. Doutora em Ciências pelo Programa de Enfermagem em Saúde Pública da Escola de Enfermagem de Ribeirão Preto da Universidade de São Paulo - EERP/USP. Professora Adjunta do Colegiado do Curso de Enfermagem da Universidade Estadual do Oeste do Paraná - UNIOESTE, campus de Cascavel. E-mail: lb.toso@certto.com.br

${ }^{5}$ Enfermeira. Doutora em Enfermagem, docente do Curso de Graduação e do Programa de Pós-Graduação em Enfermagem da Universidade Federal da Paraíba. E-mail: neucollet@gmail.com
} 
origin and care of diseases and community orientation on recognizing the needs in the community through epidemiological data and direct contact. The aim of the study was to evaluate the principles of family and community orientation of primary child health in primary health care units in Londrina, Paraná. A descriptive, quantitative, held on 39 basic units of the urban area, between August 2012 and January 2013 through an evaluation tool called PCATool Brazil-child version. A total of 609 interviews were answered by parents or primary caregivers of children under twelve, who used the service at least three times for consultation with a pediatrician or nurse. The score value for family counseling was 5.082 and community orientation 5.462, not reaching the ideal score (6.6). It was concluded that both attributes need to be developed, with improvement or installation of new measures, which may assist in improving the quality of primary care of the child.

Keywods: Evaluation of health services. Primary health care. Health services.

\section{Introdução}

Para Starfield (2002), a atenção primária em saúde (APS) é o nível do sistema que atende todas as necessidades e problemas, fornecendo atenção não só a enfermidade, mas a pessoa em si e suas condições. Caracteriza-se por um conjunto de ações voltadas ao individual e ao coletivo e que abrangem a promoção, prevenção, tratamento, reabilitação e a manutenção da saúde (BRASIL, 2007).

A atenção primaria é considerada, portanto, a porta de entrada. É o primeiro contato com o sistema de saúde e responsável por organizar o cuidado à saúde dos indivíduos, suas famílias e população, buscando propor o equilíbrio entre melhorar a saúde da população e proporcionar a equidade na distribuição de recursos (STARFIELD, 2002).

Starfield (2002, p. 58) definiu quatro atributos considerados como essenciais à atenção primária: Acesso de primeiro contato, definido como "acessibilidade e utilização do serviço a cada novo problema ou novo episódio de um problema"; Longitudinalidade: "existência de uma fonte continuada de atenção e sua utilização ao longo do tempo, além da formação de vínculos com fortes relações interpessoais entre a população e a fonte de atenção"; Integralidade: garantia de que o paciente receba todos os tipos de serviço de atenção primária, ou encaminhamento para serviços secundários e terciários, além disso, a equipe deve identificar os problemas e fornece os serviços adequados resolvêlos; e Coordenação: garante continuidade no tratamento pelos profissionais e/ou dos prontuários, fazendo com que na próxima consulta haja informação dos problemas do paciente e condutas tomadas anteriormente.

Além destes quatro atributos Starfield (2002, p. 64) elenca três derivados: a orientação familiar: "conhecimento dos fatores familiares relacionados à origem e aos cuidados das enfermidades"; orientação para a comunidade: "conhecimento do profissional sobre as necessidades da comunidade através de dados epidemiológicos e do contato com a comunidade, sua relação com ela, assim como o planejamento e a avaliação conjunta dos serviços" e; competência cultural: reconhecimento das necessidades especiais que são baseadas em diferentes culturas.

Sendo assim, um serviço de atenção básica dirigido à população pode ser considerado provedor de atenção primária quando apresentam os quatro atributos essenciais, aumentando seu poder de interação com os indivíduos e com a comunidade ao apresentar, também, os atributos derivados (HARZHEIM et al., 2006). Avaliar a efetividade da APS nos ajudará a perceber como os serviços de saúde estão em seu processo de atenção, além de contribuir para orientá-los no cumprimento dos atributos e, assim, ser capaz de promover atenção integral à sua comunidade (HARZHEIM et al., 2006).

O cuidado com a criança na atenção primária envolve também o ambiente familiar e comunitário em que ela está inserida. A família é a unidade de 
cuidado que tem grande importância na saúde e bem estar de seus membros e possui melhores condições para acompanhar e ajudar no cuidado e tratamento dos processos de saúde e doença (ANGELO; BOUSSO, 2001). Deve-se, portanto incluir a família no plano de cuidados do serviço, pois ela reflete as necessidades que cada um possui (WRIGHT; LEAHEY, 2002).

A atenção primária orientada para a comunidade é uma abordagem que utiliza habilidades epidemiológicas e clínicas complementando os programas que atendem as necessidades específicas de uma população. Com isso pode-se identificar os problemas de saúde da comunidade, planejar e avaliar os resultados de programas que atendem a população (OLIVEIRA, 2012; STARFIELD, 2002).

Assim, conhecer como está a orientação das famílias e da comunidade em que a criança está inserida, auxiliará os serviços a identificarem melhor os problemas relacionados à saúde infantil. Consequentemente, contribuirá no planejamento de ações que melhorem a qualidade do atendimento, adaptando-as com a realidade e condições da família e comunidade, além de reduzir gastos futuros com hospitalizações desnecessárias.

O objetivo desta pesquisa foi avaliar os princípios da orientação familiar e comunitária, da atenção primária à saúde da criança, por meio do instrumento Primary Care Assessment Tool-Brasil (PCATool) versão criança em Unidades Básicas de Saúde do município de Londrina, Paraná.

\section{Método}

Trata-se de um estudo descritivo e de natureza quantitativa que faz parte de um projeto multicêntrico - Avaliação da efetividade da atenção primária em saúde da criança - realizado concomitantemente nas cidades de Londrina-PR, Cascavel-PR e João Pessoa-PB.

Esta pesquisa foi realizada em 39 unidades básicas de saúde (UBS) da área urbana do município de Londrina. As 14 UBS da área rural foram excluídas por não comporem o objeto de pesquisa, visto a necessidade da identificação do cuidado à criança nos serviços da área urbana.

Para as entrevistas foi utilizado um instrumento de avaliação da atenção primária validado no Brasil por Harzheim et al. (2006). O instrumento PCATool - Brasil, possui três versões: Criança, Adulto e Profissionais. Ele permite mensurar a presença e a extensão dos atributos essenciais e derivados da APS. Apresenta uma série de perguntas que são agrupadas e separadas de acordo com cada atributo. Nesse estudo foram analisados apenas os atributos orientação familiar e orientação comunitária do PCATool versão criança que correspondem, respectivamente, aos itens I e J (BRASIL, 2010).

$\mathrm{O}$ atributo Orientação Familiar é constituído por três itens: I1 - O seu/sua "médico/enfermeiro" lhe pergunta sobre suas ideias e opiniões sobre o tratamento e cuidado de sua criança?; I2 - O seu "médico/enfermeiro" já lhe perguntou sobre doenças ou problemas que existam na família de sua criança (câncer, alcoolismo, depressão)?; I3 $\mathrm{O}$ seu "médico/enfermeiro" se reuniria com outros membros da família da criança se você achasse necessário?

O atributo Orientação Comunitária é constituído por quatro itens: J1 - Alguém do "nome do serviço de saúdel ou nome médico/enfermeiro" faz visitas domiciliares?; J2 - O "nome do serviço de saúdel ou nome médico/enfermeiro" conhece os problemas de saúde importantes de sua vizinhança?; J3 - Faz pesquisas na comunidade para identificar problemas de saúde que ele deveria conhecer?; J4 - Convida membros da família a participar do Conselho Local de Saúde (Conselho Gestor/ Conselho de Usuários)?

As respostas possíveis para cada item das variáveis eram: "com certeza sim", "provavelmente sim", "provavelmente não", "com certeza não" e "não sei/não lembro". Cada resposta possui um valor, respectivamente $4 ; 3 ; 2 ; 1 ; 9$, os quais geram escores para o atributo correspondente. 
De acordo com Starfield (1992), o valor do escore considerado como satisfatório para a presença do atributo na Unidade de Saúde é de no mínimo 6,6. O instrumento foi aplicado por alunas das quatro séries do curso de graduação em Enfermagem e por uma mestranda de Enfermagem da Universidade Estadual de Londrina, nas UBS selecionadas no período de agosto de 2012 a janeiro de 2013. As perguntas foram respondidas pelos pais ou principais cuidadores das crianças menores de 12 anos, que realizaram consultas com pediatras ou enfermeiros pelo menos três vezes até o período da coleta.

Foi aplicado também, um questionário socioeconômico e demográfico para caracterização dos pais e/ou principais cuidadores das crianças, quanto a: idade, escolaridade, renda familiar e características da moradia. Foram explicados aos entrevistados os objetivos da pesquisa e, os que aceitaram participar, assinaram o Termo de Consentimento Livre e Esclarecido (TCLE), ficando uma cópia com o entrevistado e outra com o entrevistador.

A pesquisa foi desenvolvida de acordo com as normas da Resolução 196/96 do Conselho Nacional de Saúde, tendo sido aprovada pelo Comitê de Ética em Pesquisa Envolvendo Seres Humanos da Universidade Estadual de Londrina, Parecer CEP/UEL n ${ }^{\circ}$ 061/2012, em 15/05/2012, CAAE $n^{\circ}$ 01295412.2.1001.0107.

\section{Resultados}

Foram realizadas 609 entrevistas. Destas, os principais cuidadores das crianças foram as mães (78,8\%), seguido pelos avós (13,9\%). A faixa etária dos cuidadores variou entre 17 e 19 anos de idade $(6,8 \%)$ e maiores de 60 anos $(5,4 \%)$, com prevalência entre 20 e 59 anos (87,2\%). A escolaridade para $43,7 \%$ foi de até 9 anos e 50,9\% tinham entre 10 e 14 anos de estudo.

Sobre os pais das crianças, $60,3 \%$ estavam casados. Quanto à ocupação, 35,8\% das mães era do lar e $29,2 \%$ tinha trabalho formal. Em relação aos pais, $41,4 \%$ foi identificado como trabalhadores formais.

Referente à renda, 19,1\% das famílias viviam com até um salário mínimo e 73,1\% com dois a quatro salários. Os resultados mostram ainda que em 49,6\% das famílias apenas uma pessoa contribuía com a renda da casa. E, em $36,45 \%$ dos casos, quatro pessoas eram dependentes da renda familiar.

A média e o escore geral de cada item que compõe o atributo orientação familiar estão expressos na Tabela 1.

As perguntas que receberam maior pontuação foram sobre o questionamento por parte do médico sobre o histórico clínico da família e sobre a possibilidade de reunião entre o profissional da saúde com os membros da família, mostrando uma preocupação não apenas com o usuário.

Tabela 1 - Escore geral do atributo Orientação Familiar. Entrevistados (cuidadores das crianças). Londrina, 2013

\begin{tabular}{llc}
\hline \multicolumn{1}{c}{ INDICADORES } & Média & Escore \\
\hline $\begin{array}{l}\text { I1- O seu/ sua “médico/enfermeiro” lhe pergunta sobre suas } \\
\text { ideias e opiniões sobre o tratamento e cuidado de sua criança? }\end{array}$ & 2,005 & 3,350 \\
\hline $\begin{array}{l}\text { I2- O seu “médico/enfermeiro” já lhe perguntou sobre doenças } \\
\text { ou problemas que existam na família de sua criança (câncer, } \\
\text { alcoolismo, depressão)? }\end{array}$ & 3,066 & 6,887 \\
\hline $\begin{array}{l}\text { I3- O seu “médico/enfermeiro” se reuniria com outros } \\
\text { membros da família da criança se você achasse necessário? }\end{array}$ & 2,502 & 5,007 \\
\hline ESCORE GERAL & 2,525 & 5,082 \\
\hline
\end{tabular}

Fonte: Autores. 
A orientação comunitária também perguntas que compõe esse atributo receberam obteve resultados insatisfatórios, valores abaixo de 6,6. A única pergunta que atingiu recebendo no geral escore 5,462. o valor ideal foi sobre a realização de visitas Conforme apresentado na Tabela 2,todas as domiciliares $(7,057)$.

Tabela 2 - Escore geral do atributo Orientação Comunitária. Entrevistados (cuidadores das crianças). Londrina, 2013

\begin{tabular}{lcc}
\hline \multicolumn{1}{c}{ INDICADOR } & Média & Escore \\
\hline $\begin{array}{l}\text { J1- Alguém do "nome do serviço de saúde ou nome médico/ } \\
\text { enfermeiro" faz visitas domiciliares? }\end{array}$ & 3,117 & 7,057 \\
\hline J2 - O “nome do serviço de saúde lou nome médico/enfermeiro" & & \\
conhece os problemas de saúde importantes de sua vizinhança? & 2,907 & 6,357 \\
\hline J3 - Faz pesquisas na comunidade para identificar problemas de saúde & & \\
que ele deveria conhecer? & 2,629 & 5,430 \\
\hline $\begin{array}{l}\text { J4 - Convida membros da família a participar do Conselho Local de } \\
\text { Saúde (Conselho Gestor/Conselho de Usuários)? }\end{array}$ & 1,901 & 3,003 \\
\hline ESCORE GERAL & 2,639 & 5,462 \\
\hline
\end{tabular}

Fonte: Autores.

\section{Discussão}

Os resultados obtidos são semelhantes a outros estudos. Em duas pesquisas (ELIAS, 2006; VAN STRALEN, 2008) que compararam a opinião dos usuários e dos profissionais em serviços com a Estratégia Saúde da Família (ESF) e unidades tradicionais, mostraram que para esses dois atributos os valores dos escores, foram naquelas com a ESF, mas os valores dos escores para orientação familiar e comunitária foram inferiores ao escore considerado ideal $(>6,6)$.

Vale lembrar que o primeiro estudo avaliou o município de São Paulo onde $45 \%$ das UBS possuía ESF. Já o segundo, foi realizado em vários municípios de Goiás cuja cobertura de ESF variou de $7 \%$ a $34,5 \%$. Existindo então uma grande diferença no quantitativo populacional e na cobertura de ESF quando comparados ao município de Londrina (55\%), onde não há a divisão de serviços. A justificativa usada para os autores é que o instrumento aplicado aos usuários privilegia a atuação de alguns profissionais, principalmente médicos. Os Agentes Comunitários de Saúde (ACS) são incluídos indiretamente apenas nas questões sobre orientação comunitária (ELIAS, 2006; VAN STRALEN, 2008).

Segundo Leão et al. (2011) as ESF tendem a proporcionar um modelo de assistência diferenciado que valoriza o contexto de vida das pessoas e o indivíduo inserido na família e comunidade, o que pode justificar que em seu estudo, os participantes também atribuíram melhores escores para as equipes de ESF. Resultados semelhantes foram encontrados na pesquisa de Oliveira (2007) as UBS tradicionais, não foram avaliadas como modelo para o fortalecimento das APS e a utilização da ESF aumentou a prevalência do maior escore.

Analisando as perguntas que compõem os atributos do presente estudo, a orientação familiar teve apenas um item que recebeu valor satisfatório (I2), o qual se refere às perguntas referentes aos profissionais se solicitavam informações sobre doenças e problemas presentes nas famílias, como exemplo o câncer, alcoolismo, depressão, hipertensão, diabetes e outras doenças de influência genética, mostrando preocupação com a 
possiblidade de doenças futuras que as crianças poderiam desenvolver, mas o escore foi inferior a 6,6 .

Pesquisa realizada por Oliveira (2012) também evidenciou escore inferior ao ideal. No entanto, do mesmo modo que os outros estudos mencionados (ELIAS, 2006; VAN STRALEN, 2008), ao comparar os serviços com e sem a ESF, essa pergunta também recebeu melhor escore nas unidades com ESF $(6,05)$ do que nas UBS tradicionais $(2,87)$. Isso pode ser explicado pelo fato de que os familiares nunca tiveram necessidade de fazer uma reunião ou até mesmo por não conhecerem esse direito, visto que os profissionais não têm sugerido e/ou informado sobre essa possibilidade de interação serviço comunidade.

O atributo participação dos cuidadores no tratamento das crianças (I1) também apresentou escore relativamente baixo. Isso remete para a possibilidade de que, em muitos casos, o contexto familiar não está sendo levado em conta no atendimento à criança. Segundo Elias et al. (2006), a família ainda não tem sido incorporada na assistência à saúde, sendo necessária ênfase e melhoria das ações com enfoque na abordagem dos modos de vida familiares.

O resultado para a orientação comunitária, assim como o obtido por Oliveira (2012), foi favorecido pelas visitas domiciliares, as quais mostraram a boa atuação dos profissionais, principalmente dos ACS, que são os principais responsáveis por essa tarefa. Nas equipes com ESF o escore atingiu o valor ideal $(7,57)$, e as unidades tradicionais obtiveram resultado extremamente baixo $(1,16)$.

De acordo Leão et al. (2011), alguns fatores parecem estar relacionados com a melhor efetividade da orientação comunitária na ESF, como as ações de visita domiciliar que são realizadas por todos os profissionais, principalmente pelos agentes comunitários. Fato que facilita a vigilância à saúde e o acompanhamento das famílias da comunidade.
O ACS é um personagem-chave do Programa de Agentes Comunitários de Saúde (PACS), vinculado à Unidade de Saúde da Família (USF). Ele liga a equipe à comunidade, destacando-se pela comunicação com as pessoas e pela liderança natural. É um elo cultural do Sistema Único de Saúde (SUS) com a população e, seu contato permanente com as famílias, facilita o trabalho de vigilância e promoção da saúde (BRASIL, 2006).

Convém destacar que a visita domiciliar é um instrumento usado para que os serviços consigam se aproximar e identificar as necessidades das famílias dentro da comunidade, auxiliando nas medidas que devem ser tomadas (FRACOLLI; BERTOLOZZI, 2001).

Quanto ao resultado da orientação comunitária se os usuários profissionais conheciam os problemas da comunidade, no presente estudo, atingiu escore de 6,357. Já o valor do escore sobre a realização de pesquisas na comunidade para se identificar seus problemas foi baixo $(5,430)$. Podese inferir que as pessoas não têm conhecimento sobre tais pesquisas por não serem feitas de maneira explícitas, ou seja, não apresentam um perfil de pesquisa, com perguntas estruturadas por exemplo. Elas podem estar subtendidas e sendo realizadas durante as visitas, sem que as pessoas estejam ciente de sua participação.

$\mathrm{O}$ pior escore foi o referente à participação da população nos Conselhos de Saúde. A participação nos conselhos serve para que os setores da população realizem discussões, propostas de intervenções e compartilhamento de problemas e soluções junto aos trabalhadores da saúde (FRACOLLI; BERTOLOZZI, 2001). Oliveira (2012) argumenta que a participação dos usuários pode estar sendo restrita ou que a população desconhece a existência dos Conselhos. Pode-se supor ainda, que os serviços não divulguem aos usuários esta possibilidade e, consequentemente, não estejam utilizando esta estratégia de vinculação entre serviço e comunidade. 


\section{Conclusão}

Apesar de Londrina possuir uma cobertura de $55 \%$ da ESF e seu foco principal ser a família e, consequentemente, a comunidade, os resultados obtidos indicaram que a oferta de serviços na atenção primária à saúde precisa ser aprimorada para cumprir os atributos da orientação familiar e da orientação para a comunidade.

Conclui-se que ambos os atributos favorecem a qualidade da assistência prestada pelos profissionais da atenção primária à criança mediante intervenções juntamente com os familiares, bem como interlocução comunitária para que promover ações conjuntas para transformação do meio onde vivem que, em geral, pode determinar em maior ou menor grau a forma como a população adoece e morre.

\section{Agradecimentos}

À Universidade Estadual de Londrina por fornecer a Bolsa de Iniciação Científica.

\section{Referências}

ANGELO, M.; BOUSSO, R. S. Fundamentos da assistência à família em saúde. In: BRASIL. Ministério da Saúde. Instituto para o Desenvolvimento da Saúde. Universidade de São Paulo. Manual de enfermagem. Brasília: Ministério da Saúde, 2001.p. 9-13.

BRASIL. Ministério da Saúde. Entendendo o SUS. Brasília, 2006.

Ministério da Saúde. Manual do instrumento de avaliação da atenção primária à saúde: primary care assessment tool pcatool - Brasil. Brasília, 2010.

. Ministério da Saúde. Politica Nacional de Atenção Básica. 4. ed. Brasília, 2007.

ELIAS, P. E.; FERREIRA, C. W.; GÓIS, M. C. A.; COHN, A.; KISHIMA, V.; ESCRIVÃO JUNIOR, Á.; GOMES, A.; BOUSQUAT, A. Atenção básica em saúde: comparação entre PSF e UBS por estrato de exclusão social no município de São Paulo. Ciência \& Saúde Coletiva, Rio de Janeiro, v. 11, n. 3, p. 633-641, 2006.
FRACOLLI, L. A; BERTOLOZZI, M. R. A abordagem do processo saúde-doença das famílias e do coletivo. In: BRASIL. Ministério da Saúde. Instituto para o Desenvolvimento da Saúde. Universidade de São Paulo. Manual de Enfermagem. Brasília, 2001. p. 4-8.

HARZHEIM, E.; STARFIELD, B.; RAJMIL, L.; ÁlVAREZ-DARDET, C.; STEIN, A. T. Consistência interna e confiabilidade da versão em português do instrumento de avaliação da atenção primária (PCATool-Brasil) para serviços de saúde infantil. Cadernos de Saúde Pública, Rio de Janeiro, v. 22, n. 8, p. 1649-1659, 2006.

LEÃO, C. D. A; CALDEIRA, A. P.; OLIVEIRA, M. M. C. Atributos da atenção primária na assistência à saúde da criança: avaliação dos cuidadores. Revista Brasileira de Saúde Materno Infantil, Recife, v. 11, n. 3, p. 323-334, 2011.

OLIVEIRA, M. M. C. Presença e extensão dos atributos da atenção primária á saúde nos serviços de atenção primária de Porto Alegre: uma análise agregada. 2007. Dissertação (Mestrado em Epidemiologia) - Programa de Pós-Graduação em Epidemiologia, Universidade Federal do Rio Grande do Sul, Porto Alegre.

OLIVEIRA, V. B. C. A. Avaliação da atenção primária à saúde da criança no município de Colombo -PR. 2012. Dissertação (Mestrado em Ciências) - Escola de Enfermagem, Universidade de São Paulo, São Paulo.

STARFIELD, B. Atenção primária: equilíbrio entre necessidades de saúde, serviços e tecnologia. Brasília: UNESCO/ministério da saúde, 2002.

- Primary care: concept, evaluation and policy. New York: Oxford University Press; 1992.

VAN STRALEN, C.J. BELISÁRIO, S. A.; VAN STRALEN, T. B. S.; LIMA, Â. M. D.; MASSOTE, A. W; OLIVEIRA, C. Di L. Percepção dos usuários e profissionais de saúde sobre atenção básica: comparação entre unidades com e sem saúde da família na Região Centro-Oeste do Brasil. Cadernos de Saúde Pública, Rio de Janeiro, v. 24, n. 1, p. 148158, 2008. 
WRIGHT, L. M; LEAHEY, M. Enfermeiras $e$ familias: um guia para avaliação e intervenção na família. São Paulo: Roca, 2002. 